\title{
Maricopa County's use of NSSP ESSENCE to detect cases during a hepatitis A outbreak
}

\author{
Rasneet S. Kumar*, James Matthews, Jessica R. White, Alice Carrigan, \\ Jennifer Collins, Nicole Fowle and Jigna Narang
}

Maricopa County Department of Public Health, Office of Epidemiology, Phoenix, AZ, USA

\section{Objective}

To demonstrate the utility of the National Syndromic Surveillance Program's (NSSP) version of the Electronic Surveillance System for Early Notification of Community-based Epidemics (ESSENCE) for case detection during a 2017 outbreak of hepatitis A virus (HAV) infection among persons experiencing homelessness in Maricopa County, Arizona

\section{Introduction}

On 3/29/2017, the Maricopa County Department of Public Health (MCDPH) received three reports of confirmed HAV infection from an onsite clinic at Campus A that assists individuals experiencing homelessness, a population at risk for HAV transmission. To identify the scope of the problem, the department initiated rapid HAV infection case detection using NSSP ESSENCE.

\section{Methods}

MCDPH created a myESSENCE dashboard that searched for chief complaint keywords and discharge diagnosis codes from 15 Maricopa County emergency department and inpatient hospital records using three separate queries: (1) HAV infection; (2) hepatitis virus infection; and (3) HAV infection symptoms combined with terms for homelessness. The dashboard was reviewed retrospectively for the 90 days prior to the initial report of the cluster (12/28/2016 - 3/29/2017). Based on this review, MCDPH epidemiologists decided to use the first query (HAV infection-specific) only, because reviewing the line list for all three queries was resource-intensive and resulted in duplicate cases. The query was monitored every weekday morning from $3 / 30 / 2017$ to the close of the outbreak on 7/22/2017.

When a potential HAV infection case was identified in ESSENCE, epidemiologists attempted to identify the patient in Arizona's Medical Electronic Disease Surveillance Intelligence System (MEDSIS) using date of birth. Potential cases with an existing HAV infection, MEDSIS record, and a listed address matching Campus A were interviewed by a disease investigator. When potential cases did not have a HAV infection report in MEDSIS, a review of the medical record or follow-up with the facility's infection preventionist was conducted to confirm HAV infection diagnosis and homelessness status before contacting the patient for interview. Following patient interview, all cases with a positive HAV immunoglobulin M antibody, HAV infection symptomology, and association with Campus A, were considered outbreak cases. All other patients were considered unrelated to the outbreak.

$\mathrm{MCDPH}$ evaluated the timeliness of reporting by comparing the date when outbreak cases were first available in ESSENCE to the date they were entered into MEDSIS.

\section{Results}

From 3/30/2017 - 7/22/2017, MCDPH identified 37 potential HAV infection cases in ESSENCE. Eleven cases were classified as outbreak cases, while the other 26 patients lacked recent HAV infection symptoms, laboratory confirmation, or association with Campus A. All 11 outbreak cases' records included the ICD-CM-10 code B15.9 (Hepatitis A without hepatic coma), and 3 records included the code
Z59 (Problems related to housing and economic circumstances). The HAV infection-specific query in ESSENCE identified 11 (73\%) of the 15 total outbreak cases; however, all cases were reported in MEDSIS prior to being flagged in ESSENCE. On average, cases were reported to MEDSIS 9 days earlier than identified in ESSENCE (range, 0 - 21 days). Of note, ESSENCE helped identify the outbreak index case, previously lost to follow-up, as an individual experiencing homelessness. This information helped MCDPH perform additional follow-up, which revealed that the individual had arrived from San Diego, CA, a city with an ongoing outbreak of HAV infection in their homeless and drug-using population. This epidemiologic link was identified on 3/30/2017, over a month before the Centers for Disease Control and Prevention confirmed a match by genome sequencing between the index case and the neighboring outbreak on 5/5/2017.

\section{Conclusions}

Use of ESSENCE identified most of the HAV infection cases from this outbreak among individuals experiencing homelessness but no sooner than traditional surveillance methods. However, use of ESSENCE allowed for identification of the outbreak index case, leading to epidemiological linkage to outbreak origin approximately one month prior to molecular laboratory confirmation.

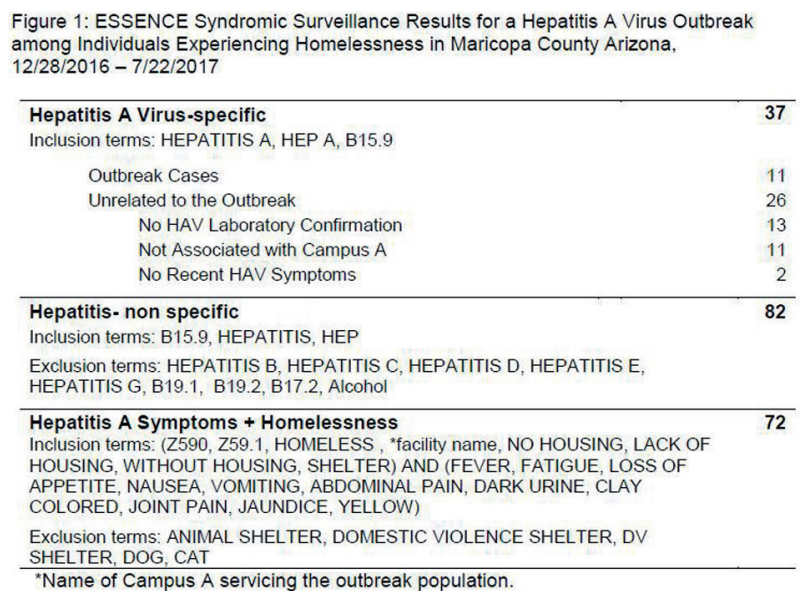

Keywords

Hepatitis A Virus; Homeless; Syndromic Surveillance; ESSENCE

${ }^{*}$ Rasneet S. Kumar

E-mail: rasneetkumar@mail.maricopa.gov 